\title{
Nonconservative electric and magnetic optical forces on submicron dielectric particles
}

\author{
Raquel Gómez-Medina, ${ }^{1, *}$ Manuel Nieto-Vesperinas, ${ }^{1}$ and Juan José Sáenz ${ }^{2,3}$ \\ ${ }^{1}$ Instituto de Ciencia de Materiales de Madrid, Consejo Superior de Investigaciones Cientificas, Campus de Cantoblanco, \\ Madrid E-28049, Spain \\ ${ }^{2}$ Departamento de Física de la Materia Condensada, Universidad Autónoma de Madrid, E-28049 Madrid, Spain \\ ${ }^{3}$ Donostia International Physics Center (DIPC), Paseo Manuel Lardizabal 4, 20018 Donostia-San Sebastian, Spain
}

(Received 30 November 2010; published 23 March 2011)

\begin{abstract}
We present a study of the total force on a small lossless dielectric particle, which presents both an electric and magnetic response, in a optical vortex wave field. We show that the force is a simple combination of conservative and nonconservative steady forces that can rectify the flow of magnetodielectric particles. In a vortex lattice the electric-magnetic dipolar interaction can spin the particles either in or out of the whirl sites leading to trapping or diffusion. Specifically, we analyze force effects on submicron silicon spheres in the near infrared, proving that the results previously discussed for hypothetical magnetodielectric particles can be observed for these $\mathrm{Si}$ particles.
\end{abstract}

DOI: 10.1103/PhysRevA.83.033825

PACS number(s): 42.25.Bs, 42.25.Fx, 42.25.Gy, 41.20.Jb

\section{INTRODUCTION}

The understanding and control of particle transport and diffusion properties is a most relevant issue in fields ranging from biophysics to material science and chemical processing, with countless applications which include particle mixing, diffusive separation of particles, microrhelogy, or intracellular transport or drug delivery, to mention a few [1-4]. The advances in sculpting optical wavefronts and light intensity profiles make optical tools ideal for both imaging and manipulation of particles. Optical fields are easily tunable in general and affect any polarizable object, from atoms to microscopic colloidal particles $[5,6]$. These fields can be used to arrange, guide or deflect particles in appropriate light pattern geometries [7-11]. Intense optical waves can also induce significant forces between particles [12-17].

Light forces on small dielectric particles are traditionally described as the sum of two terms: the dipole or gradient force and the radiation pressure or scattering force proportional to the Poynting vector [18-20]. A nonconservative scattering curl force appears when the spatial distribution of the field polarization is not uniform [21]. For magnetodielectric particles [22,23], the force presents both electric and magnetic gradient and scattering contributions together with an additional term due to the electric-magnetic dipolar interaction, that contributes to both the scattering force and to the gradient force [23]. The main purpose of this work is to illustrate the relevance of this additional contribution in the particularly simple case of a two-dimensional (2D) field geometry, arising at the intersection region of two standing plane waves.

In free space, the calculation of optical forces acting on small (Rayleigh) dipolar particles in two-dimensional optical lattices is relatively simple, allowing analytical treatment of the problem $[18,21,24-26]$. Even in this simple case, the particle dynamics in an optical vortex lattice (arising in the intersection region of crossed optical standing waves [27]) presents a number of interesting properties $[25,26]$. However, when the particle size is of the order of or larger than the internal

\footnotetext{
*rgomezmedina@icmm.csic.es
}

wavelength, in the so-called "Mie" regime, it is difficult to obtain systematic predictions and most theoretical work in this regime is based on a numerical approach [28]. An analytical approach for the optical forces on particles far beyond the Rayleigh limit is still possible provided the scattering can be described by the first two electric and magnetic Mie coefficients [23]. Here we discuss the strong magnetic and electric optical forces on submicron dielectric particles with unusual scattering effects. As we will show, the electricmagnetic dipolar interaction plays an active role in spinning the particles either in or out of the whirl sites of the interference pattern, leading to trapping or diffusion. Specifically, optical forces, previously discussed for hypothetical magnetodielectric particles [23], are now discussed for submicron silicon spheres, whose magnetodielectric properties in the near infrared have been recently pointed out [29]. In contrast with typical (relatively low-index) polystyrene particles, Si particles present strong magnetic and electric resonant scattering cross sections and their scattering properties are well described by its first two electric and magnetic Mie coefficients [29]. To illustrate that they provide a real example and unique laboratory to explore nonconservative electric and magnetic optical forces is another of the main outcomes of this work.

Finally, we show that a simple combination of conservative and nonconservative steady forces can rectify the flow of magnetodielectric particles. This may permit the exploration of new forms of controlled atom motion in optical lattices $[27,30]$ and may be used to separate and sort small particles with slightly different optical characteristics $[10,11]$.

\section{FORCE ON A SMALL PARTICLE WITH ELECTRIC AND MAGNETIC RESPONSE IN AN OPTICAL VORTEX FIELD}

Let us consider a magnetodielectric particle immersed in a medium with real relative dielectric permittivity $\epsilon$ and magnetic permeability $\mu$, illuminated by a time-harmonic electromagnetic field $\mathbf{E}(\mathbf{r}) e^{-i \omega t}, \mathbf{B}(\mathbf{r}) e^{-i \omega t}$ with wave number $k=n \omega / c(c$ is the speed of light in vacuum, $\omega$ is the frequency, and $n=\sqrt{\epsilon \mu}$ stands for the refractive index of the surrounding medium). Thus we shall concentrate on the space-dependent 
part of the wave field from now on. The time-averaged force on a dipolar magnetodielectric particle, characterized by its electric and magnetic polarizabilities $\alpha_{\mathrm{e}}$ and $\alpha_{\mathrm{m}}$, is given by the sum of three terms [23]: $\langle\mathbf{F}\rangle=\left\langle\mathbf{F}_{\mathrm{e}}\right\rangle+\left\langle\mathbf{F}_{\mathrm{m}}\right\rangle+\left\langle\mathbf{F}_{\mathrm{em}}\right\rangle$. The "electric force" $\left\langle\mathbf{F}_{\mathrm{e}}\right\rangle$ corresponds to the standard optical force due to the induced electric dipole [18] and can be written as [21]

$$
\left\langle\mathbf{F}_{e}\right\rangle=\frac{4 \pi \operatorname{Re}\left\{\alpha_{\mathrm{e}}\right\}}{\epsilon} \nabla\left\langle U_{\mathrm{e}}\right\rangle+\sigma_{\mathrm{e}}^{\mathrm{ext}}\left\{\frac{n}{c}\langle\boldsymbol{S}\rangle\right\}-\sigma_{\mathrm{e}}^{\mathrm{ext}}\left\{\frac{c}{n} \nabla \times\left\langle\boldsymbol{L}_{S \mathrm{e}}\right\rangle\right\},
$$

where $\sigma_{\mathrm{e}}^{\mathrm{ext}}=4 \pi k \operatorname{Im}\left[\epsilon^{-1} \alpha_{\mathrm{e}}\right]$ is the electric extinction cross section and

$$
\begin{gathered}
\left\langle U_{\mathrm{e}}\right\rangle=\frac{1}{2} \frac{\epsilon}{8 \pi}|\mathbf{E}|^{2}, \\
\langle\boldsymbol{S}\rangle=\frac{1}{2} \frac{c}{4 \pi \mu} \operatorname{Re}\left\{\mathbf{E} \times \mathbf{B}^{*}\right\}, \\
\left\langle\boldsymbol{L}_{S \mathrm{e}}\right\rangle=\frac{1}{2} \frac{\epsilon}{8 \pi \omega i} \mathbf{E}^{*} \times \mathbf{E},
\end{gathered}
$$

are the time-average electric energy density, Poynting vector, and electric spin density of the optical field, respectively.

The first, second, and third terms of the right-hand side of Eq. (1) corresponds to the gradient force $\left(\left\langle\mathbf{F}_{\mathrm{e}}^{\langle U\rangle}\right\rangle\right)$, radiation pressure $\left(\left\langle\mathbf{F}_{\mathrm{e}}^{\langle S\rangle}\right\rangle\right)$, and electric spin $\left(\left\langle\mathbf{F}_{\mathrm{e}}^{\langle L\rangle}\right\rangle\right)$ forces, respectively [21]. The force $\left\langle\mathbf{F}_{\mathrm{m}}\right\rangle$ on the induced magnetic dipole has an analogous decomposition as the former [23] in terms of a gradient component, a radiation pressure or scattering component (which is the only one contributing in the case of a plane wave), and a third term that depends on the polarization of the magnetic field [analogously to its electric counterpart Eq. (4)]; namely,

$$
\begin{aligned}
\left\langle\mathbf{F}_{\mathrm{m}}\right\rangle= & \left\langle\mathbf{F}_{\mathrm{m}}^{\langle U\rangle}\right\rangle+\left\langle\mathbf{F}_{\mathrm{m}}^{\langle S\rangle}\right\rangle+\left\langle\mathbf{F}_{\mathrm{m}}^{\langle L\rangle}\right\rangle=\frac{4 \pi \operatorname{Re}\left\{\alpha_{\mathrm{m}}\right\}}{\epsilon} \nabla\left\langle U_{\mathrm{m}}\right\rangle \\
& +\sigma_{\mathrm{m}}^{\operatorname{ext}}\left\{\frac{n}{c}\langle\boldsymbol{S}\rangle\right\}-\sigma_{\mathrm{m}}^{\operatorname{ext}}\left\{\frac{c}{n} \nabla \times\left\langle\boldsymbol{L}_{S \mathrm{~m}}\right\rangle\right\}
\end{aligned}
$$

where $\sigma_{\mathrm{m}}^{\mathrm{ext}}=4 \pi k \operatorname{Im}\left[\mu \alpha_{\mathrm{m}}\right]$ is the magnetic extinction cross section and

$$
\begin{gathered}
\left\langle U_{\mathrm{m}}\right\rangle=\frac{1}{2} \frac{1}{8 \pi \mu}|\mathbf{B}|^{2}, \\
\left\langle\boldsymbol{L}_{S \mathrm{~m}}\right\rangle=\frac{1}{2} \frac{1}{8 \pi \mu \omega i} \mathbf{B}^{*} \times \mathbf{B} .
\end{gathered}
$$

Finally, the interaction between electric and magnetic dipoles $[22,23]$ leads to an additional force $\left\langle\mathbf{F}_{\mathrm{em}}\right\rangle$ given by [23]

$$
\begin{aligned}
\left\langle\mathbf{F}_{\mathrm{em}}\right\rangle= & \left\langle\mathbf{F}_{\mathrm{em}}^{\langle S\rangle}\right\rangle+\left\langle\mathbf{F}_{\mathrm{em}}^{\langle U, L\rangle}\right\rangle=-\frac{8 \pi}{3} k^{4} \frac{\mu}{\epsilon} \operatorname{Re}\left(\alpha_{\mathrm{e}} \alpha_{\mathrm{m}}^{*}\right)\left\{\frac{n}{c}\langle\boldsymbol{S}\rangle\right\} \\
& +\frac{k^{4}}{3} \sqrt{\frac{\mu}{\epsilon}} \operatorname{Im}\left(\alpha_{\mathrm{e}} \alpha_{\mathrm{m}}^{*}\right)\left\{\operatorname{Im}\left(\mathbf{E} \times \mathbf{B}^{*}\right)\right\}
\end{aligned}
$$

\section{A. Forces in an optical vortex lattice}

Let us now consider the electric field in the interference region of two standing plane waves oriented along the $x$ and $y$

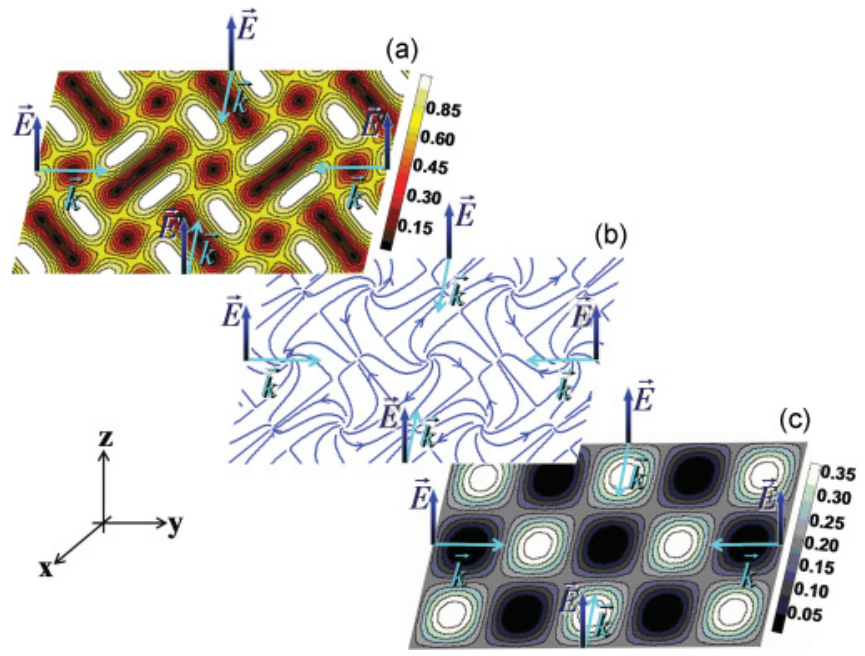

FIG. 1. (Color online) Illustration of the maps of (a) the total force modulus, (b) the force lines and (c) electric field intensity for a dipolar magnetodielectric particle in the intersection region of two standing plane waves oriented along the $x$ and $y$ axes and with the electric field polarized along the $z$ axis.

axes and with the electric field polarized along the $z$ axis:

$$
\begin{aligned}
E_{z}(x, y) & =\frac{E_{0}}{\sqrt{2}}\left(\left\{e^{i k x}-e^{-i k x}\right\}+e^{i \phi}\left\{e^{i k y}-e^{-i k y}\right\}\right) \\
& =\frac{2 i E_{0}}{\sqrt{2}}\left(\sin k x+e^{i \phi} \sin k y\right)
\end{aligned}
$$

where $\phi$ is the phase shift between the two beams. Figure 1 illustrates this geometry by showing three generic distributions corresponding to the modulus of the resulting total force [Fig. 1(a)], the force lines [Fig. 1(b)], and the electric field intensity [Fig. 1(c)].

Since the electric field polarization is constant, the electric spin forces are zero (i.e., $\left\langle\mathbf{F}_{\mathrm{e}}^{\langle L\rangle}\right\rangle=0$ ). In contrast, the polarization of the magnetic field is not constant. It can be shown (see Appendix) that the magnetic spin contribution exactly cancels the magnetic radiation pressure term (i. e., $\left\langle\mathbf{F}_{\mathrm{m}}^{\langle S\rangle}\right\rangle=-\left\langle\mathbf{F}_{\mathrm{m}}^{\langle L\rangle}\right\rangle$ ). Then the total force can be expressed as the sum of conservative "gradient" forces and nonconservative "curl" forces:

$$
\langle\mathbf{F}\rangle=\left\langle\mathbf{F}^{\text {grad }}\right\rangle+\left\langle\mathbf{F}^{\text {curl }}\right\rangle
$$

with $\left\langle\mathbf{F}^{\mathrm{grad}}\right\rangle=\left\langle\mathbf{F}_{\mathrm{e}}^{\langle U\rangle}\right\rangle+\left\langle\mathbf{F}_{\mathrm{em}}^{\langle U, L\rangle}\right\rangle+\left\langle\mathbf{F}_{\mathrm{m}}^{\langle U\rangle}\right\rangle$, where

$$
\begin{gathered}
\left\langle\mathbf{F}_{\mathrm{e}}^{\langle U\rangle}\right\rangle=\frac{4 \pi \operatorname{Re}\left\{\alpha_{\mathrm{e}}\right\}}{\epsilon} \nabla\left\langle U_{\mathrm{e}}\right\rangle, \\
\left\langle\mathbf{F}_{\mathrm{em}}^{\langle U, L\rangle}\right\rangle=\frac{8 \pi}{3} k^{3} \frac{\mu}{\epsilon} \operatorname{Im}\left\{\alpha_{\mathrm{e}} \alpha_{\mathrm{m}}^{*}\right\} \nabla\left\langle U_{\mathrm{e}}\right\rangle, \\
\left\langle\mathbf{F}_{\mathrm{m}}^{\langle U\rangle}\right\rangle=\frac{4 \pi \operatorname{Re}\left\{\alpha_{\mathrm{m}}\right\}}{\epsilon} \nabla\left\langle U_{\mathrm{m}}\right\rangle,
\end{gathered}
$$

and $\left\langle\mathbf{F}^{\text {curl }}\right\rangle=\left\langle\mathbf{F}_{\mathrm{e}}^{\langle S\rangle}\right\rangle+\left\langle\mathbf{F}_{\mathrm{em}}^{\langle S\rangle}\right\rangle$, where

$$
\begin{gathered}
\left\langle\mathbf{F}_{\mathrm{e}}^{\langle S\rangle}\right\rangle=\frac{4 \pi \operatorname{Im}\left\{\alpha_{\mathrm{e}}\right\}}{\epsilon}\left\{\frac{k n}{c}\langle\boldsymbol{S}\rangle\right\}, \\
\left\langle\mathbf{F}_{\mathrm{em}}^{\langle S\rangle}\right\rangle=-\frac{8 \pi}{3} k^{3} \frac{\mu}{\epsilon} \operatorname{Re}\left(\alpha_{\mathrm{e}} \alpha_{\mathrm{m}}^{*}\right)\left\{\frac{k n}{c}\langle\boldsymbol{S}\rangle\right\} .
\end{gathered}
$$


The expressions (11), (12), and (13) are proportional to the gradient of the electric and magnetic energy density, respectively; that is,

$$
\begin{gathered}
\nabla\left\langle U_{\mathrm{e}}\right\rangle= \\
\frac{\epsilon}{8 \pi}\left|E_{0}\right|^{2} \nabla\left\{\sin ^{2} k x+\sin ^{2} k y\right. \\
+2 \cos \phi \sin k x \sin k y\}, \\
\nabla\left\langle U_{\mathrm{m}}\right\rangle=-\frac{\epsilon}{8 \pi}\left|E_{0}\right|^{2} \nabla\left\{\sin ^{2} k x+\sin ^{2} k y\right\} .
\end{gathered}
$$

Equations (14) are proportional to the time-averaged Poynting vector:

$$
\left\{\frac{k n}{c}\langle\boldsymbol{S}\rangle\right\}=\frac{\epsilon}{4 \pi}\left|E_{0}\right|^{2} \nabla \times\left\{\hat{\mathbf{u}}_{z} \sin \phi \cos k x \cos k y\right\} .
$$

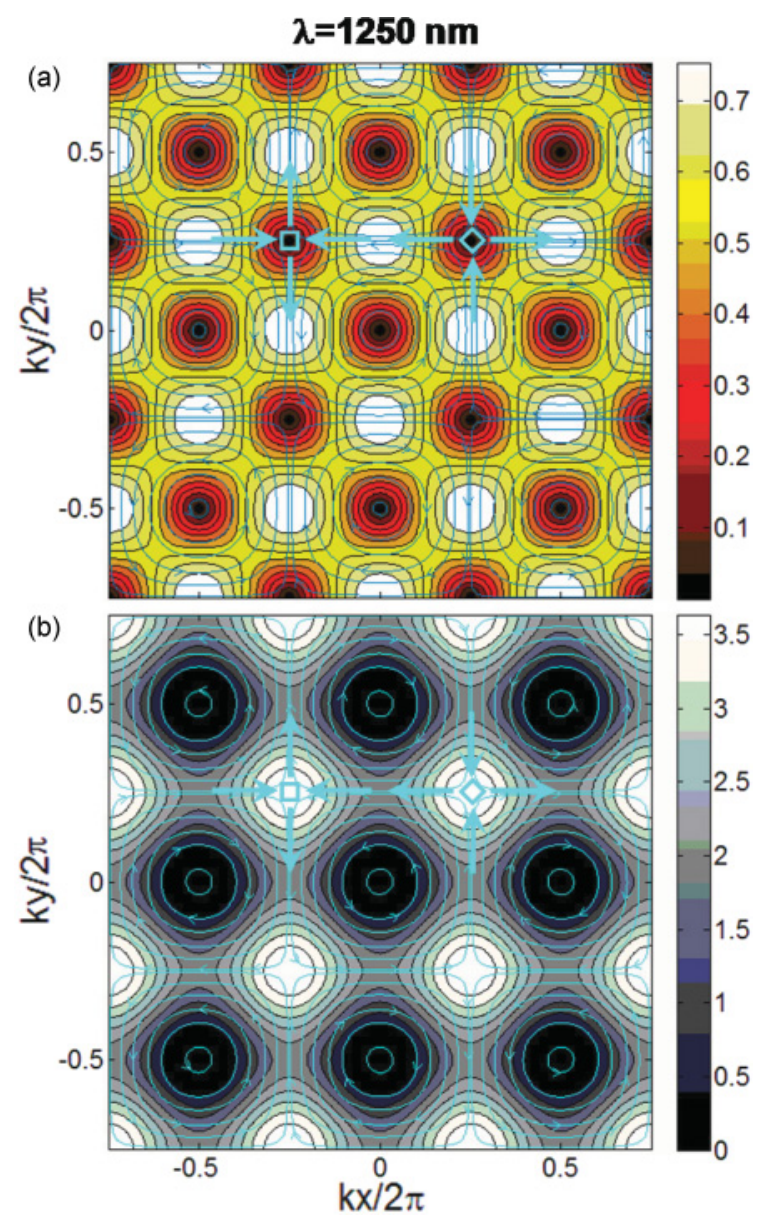

FIG. 2. (Color online) Nonconservative forces on a Si sphere of radius $a=230 \mathrm{~nm}$ placed at the intersection region of two standing waves with a dephasing $\phi=\pi / 2$ in a medium with $\epsilon=\mu=1$. The light field wavelength, $\lambda=1250 \mathrm{~nm}$, is tuned to the dipolar electric resonance. Arrows in (a) and (b) point along the total force lines. (a) Contour maps of the modulus of the normalized total force, $|\langle\mathbf{F}\rangle| / F_{0}$ with $F_{0}=\left|E_{0}\right|^{2} /\left(k a^{3}\right)$. (b) Contour maps of the normalized electric field intensity, $|\mathbf{E}|^{2} /\left|E_{0}\right|^{2}$. Near the resonance, the radiation pressure force, $\left\langle\mathbf{F}_{\mathrm{e}}^{\langle S\rangle}\right\rangle$, overcomes the attractive gradient forces and dominates the total force which presents no stable equilibrium positions. The symbols $\square$ and $\diamond$ indicate saddle points in the force map.

\section{B. Force fields and beam dephasing}

From the discussion above, notice that $\left\langle\mathbf{F}_{\mathrm{e}}\right\rangle$ is conservative or nonconservative depending on the phase, while $\left\langle\mathbf{F}_{\mathrm{m}}\right\rangle$ is always conservative (the opposite holds for $p$-polarized fields - with the magnetic field polarized in the $z$ direction). Optical forces resulting from the interference field given by Eq. (9) can be tuned by changing the phase between the beams $[25,27]$. Experimentally, this phase $\phi$ between the two crossed standing waves, can be set to any desired value produced by the optical path length difference between the beams. This may be achieved by controlling the position of one of the reflecting mirrors involved in the formation of the four mutually coherent counterpropagating beams; for example, with the two branches of a Michelson interferometer setup, as discussed in Ref. [27].

When lasers oscillate synchronously(i.e., for $\phi=0$, $\left\langle\mathbf{F}^{\text {curl }}\right\rangle=0$ ), therefore, the force is conservative. It is well known [31] that, when the electric resonance is tuned, $\operatorname{Re}\left\{\alpha_{\mathrm{e}}\right\}=0$ and $\operatorname{Im}\left\{\alpha_{\mathrm{e}}\right\}=\operatorname{Im}\left\{\alpha_{\mathrm{e}}\right\}^{\max }$ (for lossless particles $\operatorname{Im}\left\{\alpha_{\mathrm{e}}\right\} \propto \lambda^{2}$ and is independent of both particle size and material). Just at this resonance, the electric force becomes zero, $\left\langle\mathbf{F}_{\mathrm{e}}\right\rangle=\left\langle\mathbf{F}_{\mathrm{e}}^{\langle U\rangle}\right\rangle=\left\langle\mathbf{F}_{\mathrm{e}}^{\langle S\rangle}\right\rangle=0$; therefore, the total force would be zero if we did not take the magnetic and electric-magnetic interaction force into account. However, the magnetic and the interference term leads to a finite force. This should be observed in future experiments, in contrast with the previous predictions that considered only dipolar electric forces.

For $\phi=\pi / 2$ the force has contributions from the conservative and nonconservative forces. The nonconservative forces arise as a consequence of the rotation of the Poynting vector around the field nodes. In this case, when the wavelength is close to the electric dipolar resonance (i.e., $\operatorname{Re}\left\{\alpha_{\mathrm{e}}\right\} \approx 0$ and $\operatorname{Im}\left\{\alpha_{\mathrm{e}}\right\} \approx \operatorname{Im}\left\{\alpha_{\mathrm{e}}\right\}^{\max }$, , the large electric extinction cross section enhances $\left\langle\mathbf{F}_{\mathrm{e}}^{\langle S\rangle}\right\rangle$ and overcomes the attractive gradient forces so that there are no stable equilibrium positions in the system, as is the case for a metallic particle when the
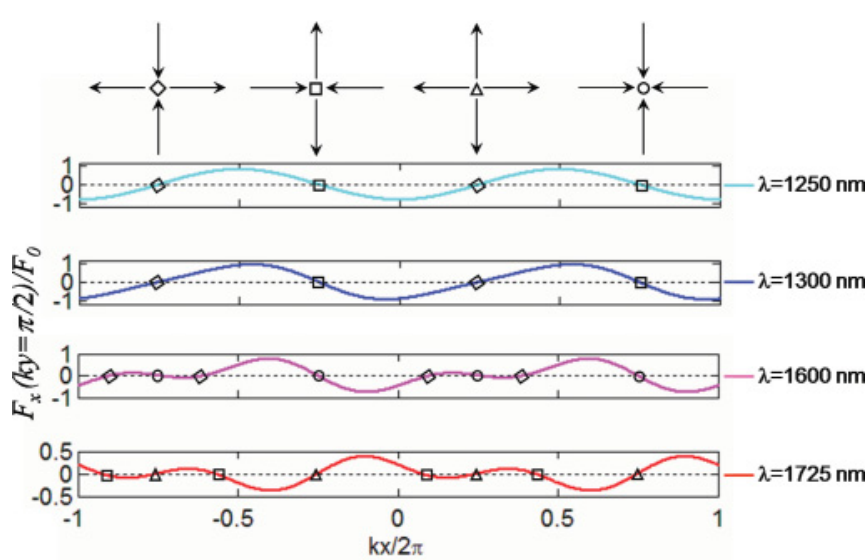

FIG. 3. (Color online) Normalized force in the $x$ direction, $F_{x}$ along a line defined by $k y=\pi / 2$ for a Si sphere of radius $a=230 \mathrm{~nm}$ in the intersection region of two standing waves with $\phi=\pi / 2$ and for different wavelengths. Notice that, since the force magnitude $F_{0}$ is inversely proportional to $k a^{3}$, this normalization factor is different for each of the four plots. The symbols sketch the force fields at several positions: $\diamond$ and $\square$ correspond to saddle points and $\triangle$ and $\bigcirc$ correspond to unstable and stable equilibrium (zero-force) positions, respectively. 
dissipation dominates [25]. Nevertheless, as the wavelength goes away from the electric resonance, a stable equilibrium position can be found due to $\left\langle\mathbf{F}_{\mathrm{e}}^{\langle S\rangle}\right\rangle$ being compensated by the rest of the force contributions.

Notice that, for $\phi=\pi / 2$, the gradient of the electric and magnetic energy density are equal except for the sign, $\nabla\left\langle U_{\mathrm{m}}\right\rangle=-\nabla\left\langle U_{\mathrm{e}}\right\rangle$. Therefore it is possible to obtain a purely nonconservative force if the particle polarizabilities fulfill $\operatorname{Re}\left\{\alpha_{\mathrm{e}}\right\}=\operatorname{Re}\left\{\alpha_{\mathrm{m}}\right\}$ and $\operatorname{Im}\left\{\alpha_{\mathrm{e}}\right\}=\operatorname{Im}\left\{\alpha_{\mathrm{m}}\right\}$ because, in this case, $\left\langle\mathbf{F}_{\mathrm{m}}^{\langle U\rangle}\right\rangle=-\left\langle\mathbf{F}_{\mathrm{e}}^{\langle U\rangle}\right\rangle,\left\langle\mathbf{F}_{\mathrm{em}}^{\langle U, L\rangle}\right\rangle=0$, and the gradient force becomes zero.

\section{OPTICAL FORCES ON SUBMICRON Si PARTICLES}

A recent work [29] shows that dielectric spheres whose refractive index is around 3.5 and whose size parameter $k a$ is between 0.75 and 1.5 are excellent instances of magnetodielectric particles whose electric and magnetic polarizabilities $\alpha_{\mathrm{e}}$ and $\alpha_{\mathrm{m}}$ are well described by

$$
\alpha_{\mathrm{e}}=i \frac{3 \epsilon}{2 k^{3}} a_{1}, \quad \alpha_{\mathrm{m}}=i \frac{3}{2 \mu k^{3}} b_{1},
$$

where $a_{1}$ and $b_{1}$ are their two Mie coefficients. In this connection, a very illustrative example to test all these new

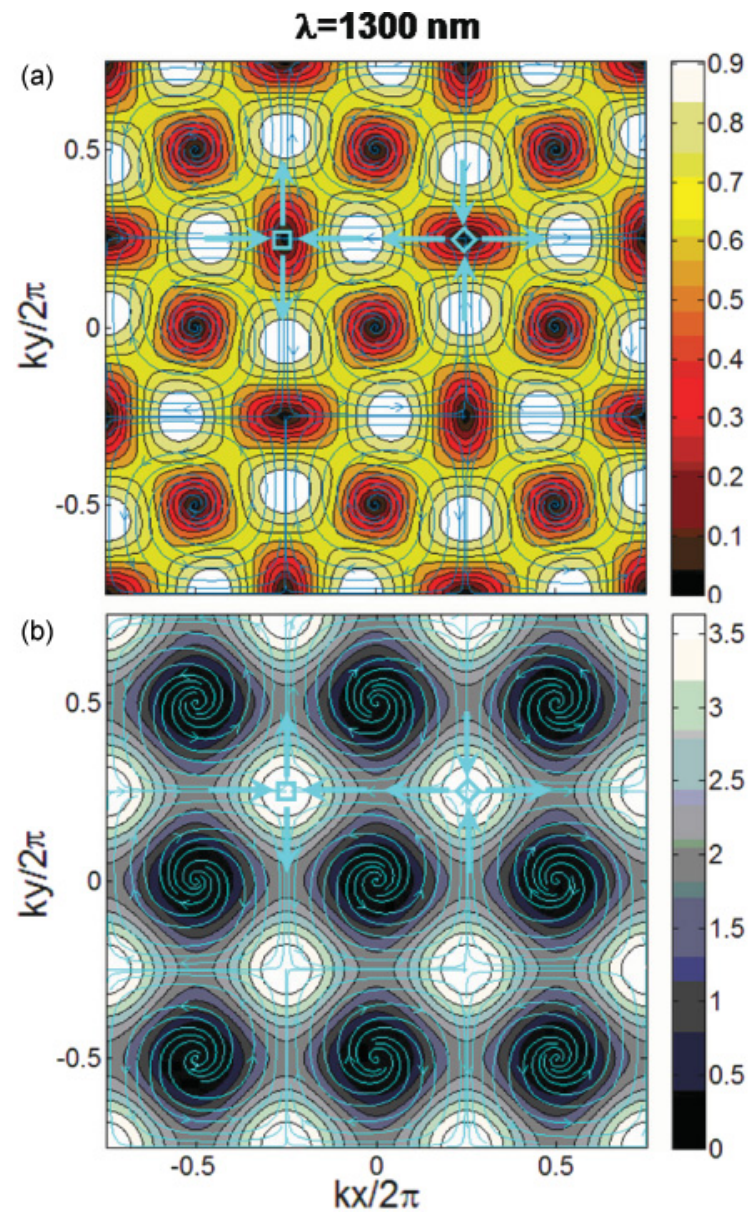

FIG. 4. (Color online) Same as Fig. 2 but for a wavelength $\lambda=$ $1300 \mathrm{~nm}$ slightly above (red-shifted) the electric dipolar resonance. The force field, dominated by the scattering force contributions, presents no stable equilibrium position in the system. results is a silicon sphere of radius $a=230 \mathrm{~nm}$ whose refractive index may well be approximated by $n_{p}=3.5$ in the range of near infrared wavelengths and the spectral line shapes of these electric and magnetic Mie resonances are well resolved with little overlap [29,32].

In the vortex lattice, each vortex is always centered around the nodes of the electric field and limited by force lines located at $k x=(2 m+1) \pi / 2$ and $k y=(2 l+1) \pi / 2$ with $m$ and $l$ integer numbers. This is independent of the light wavelength. However, the overall structure of the force field strongly depends on $\lambda$.

As an example, near the electric dipolar resonance of the $\mathrm{Si}$ particle (at $\lambda \approx 1250 \mathrm{~nm}$ ), the strong electric nonconservative component of the force, Eq. (14), induces orbital line forces around these areas where the electric field is less intense and prevents finding stable equilibrium positions in the system (see Fig. 2). Notice that, in the overdamped regime the particle flow follows the force lines [26]. Along a given line (for example, for $k y=\pi / 2$ and $-2 \pi \leqslant k x \leqslant 2 \pi)$, the zero $F_{x}$ points along $x$ (see $\lambda=1250 \mathrm{~nm}$ in Fig. 3), corresponding to saddle points in the 2D force-field map of Fig. 2.

As the wavelength increases, the dipolar electric response decreases and, for $\lambda \approx 1650 \mathrm{~nm}$, the (230-nm-radius) $\mathrm{Si}$

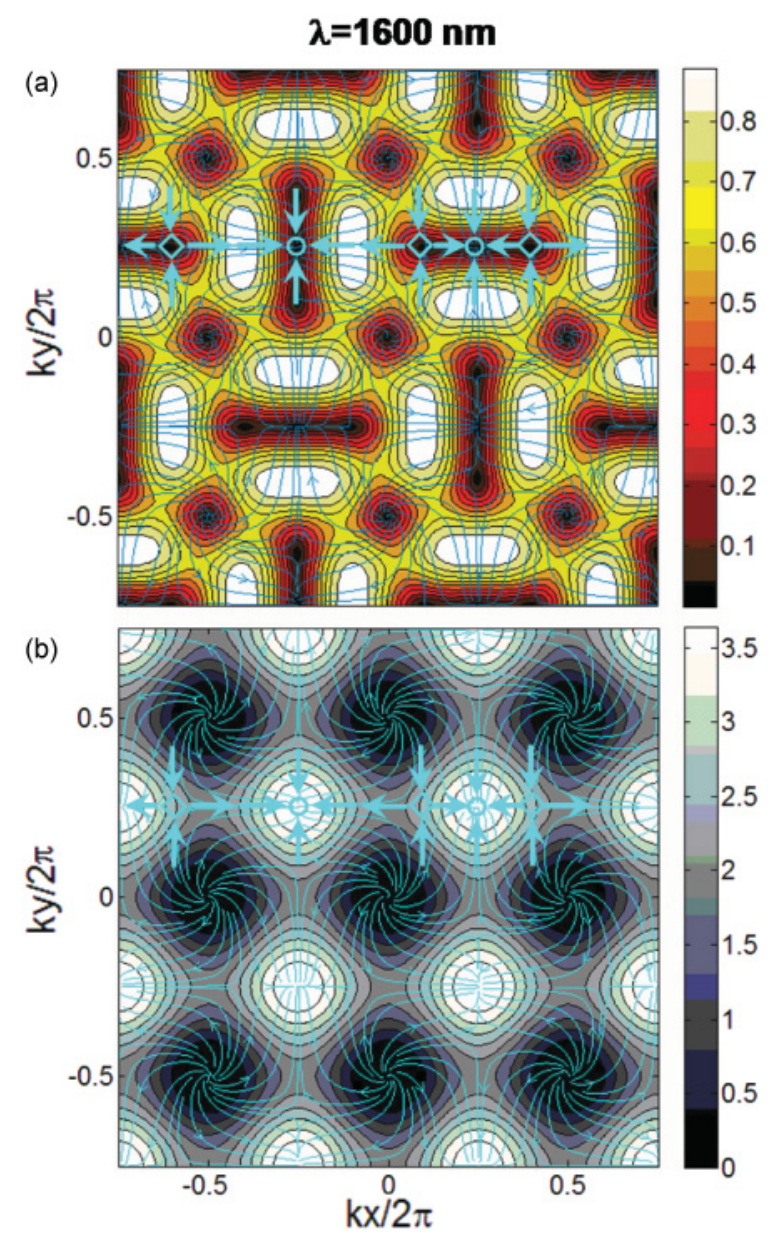

FIG. 5. (Color online) Same as Fig. 2 for a wavelength $\lambda=$ $1600 \mathrm{~nm}$ slightly below (blue-shifted) the magnetic dipolar resonance. Equilibrium (zero-force) positions correspond to electric field maxima. 
particle presents a strong magnetic resonance (see Fig. 1 in Ref. [32] and Fig. 3 in Ref. [29]). The corresponding changes in the force-field maps are illustrated in Figs. 4-7. These changes manifest themselves in the $x$ component of the force along the $k y=\pi / 2$ line, as shown in Fig. 3.

For wavelengths slightly above (red-shifted) the electric dipolar resonance, the electric polarizability is larger than zero and the contribution of the conservative gradient force is not negligible. As can be seen in Fig. 4 , for $\lambda \approx 1300 \mathrm{~nm}$ the circular "orbits" become "whirls" around the nodes of the electric field. However, gradient forces are not strong enough to overcome scattering forces and there are no stable equilibrium positions in the system. The force along the division lines still present the same "saddle" points as in resonance (Fig. 3).

Far enough from electric resonance, but still below (blue shifted) the dipolar magnetic resonance, the gradient forces dominate, giving rise to stable equilibrium positions in the system centered where the electric field is more intense because $\operatorname{Re}\left\{\alpha_{\mathrm{e}}\right\}>0$ and $\operatorname{Re}\left\{\alpha_{\mathrm{m}}\right\}<0$ so that $\left\langle\mathbf{F}_{\mathrm{e}}^{\langle U\rangle}\right\rangle$ and $\left\langle\mathbf{F}_{\mathrm{m}}^{\langle U\rangle}\right\rangle$ contribute to the conservative force with same sign [Eqs. (11) and (13)]. This is illustrated in Figs. 5 and 6 where we plotted the force map at $\lambda \approx 1600 \mathrm{~nm}$. Now the stable positions correspond to the high-intensity-field regions far from the vortex centers. This can also be seen in Fig. 3, where the original saddle points become equilibrium positions (i.e., effective potential minima) and new saddle points appear between two minima.

Above the magnetic resonance (red-shifted), $\operatorname{Re}\left\{\alpha_{\mathrm{m}}\right\}>$ $\operatorname{Re}\left\{\alpha_{\mathrm{e}}\right\}>0$ in such way that $\left\langle\mathbf{F}_{\mathrm{m}}^{\langle U\rangle}\right\rangle$ [Eq. (13)] dominates

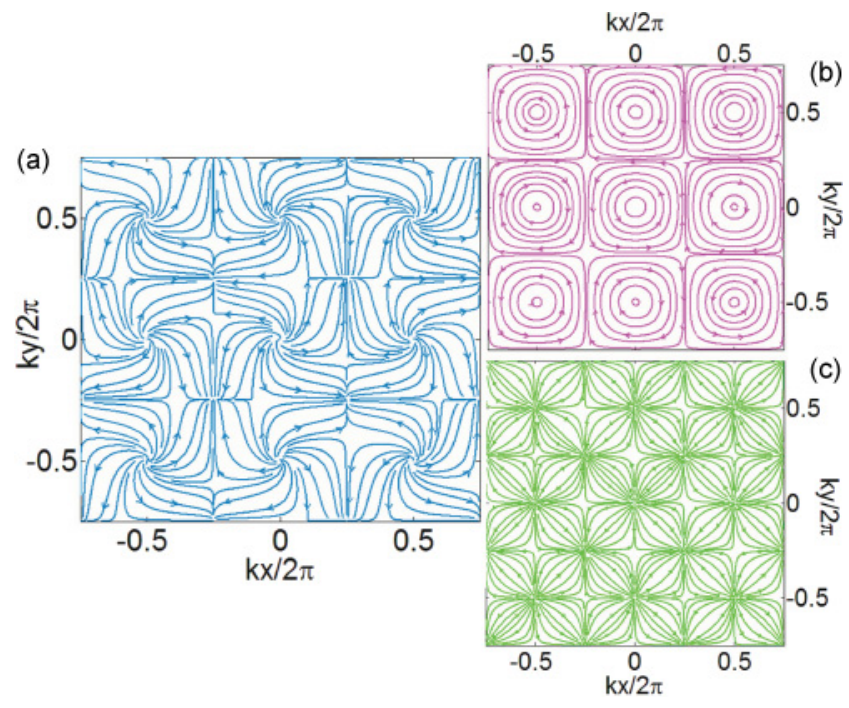

FIG. 6. (Color online) (a) Total force lines, (b) scattering force lines, and (c) conservative force lines for a $\mathrm{Si}$ sphere of radius $a=230 \mathrm{~nm}$ placed at the intersection region of two standing waves with a dephasing $\phi=\pi / 2$ in a medium with $\epsilon=\mu=1$. The light field wavelength, $\lambda=1600 \mathrm{~nm}$, is slightly below (blue-shifted) the magnetic dipolar resonance (same as Fig. 5). The rotation of the Poynting vector around the field nodes [Fig. 6(b)] induces orbital scattering force lines around these nodes; however, the conservative force [Fig. 6(c)] pushes the particle out from these nodes and toward stable positions at the corners between adjacent orbiting domains. The interplay between these two forces produces the whirls on the total forces [Fig. 6(a)].

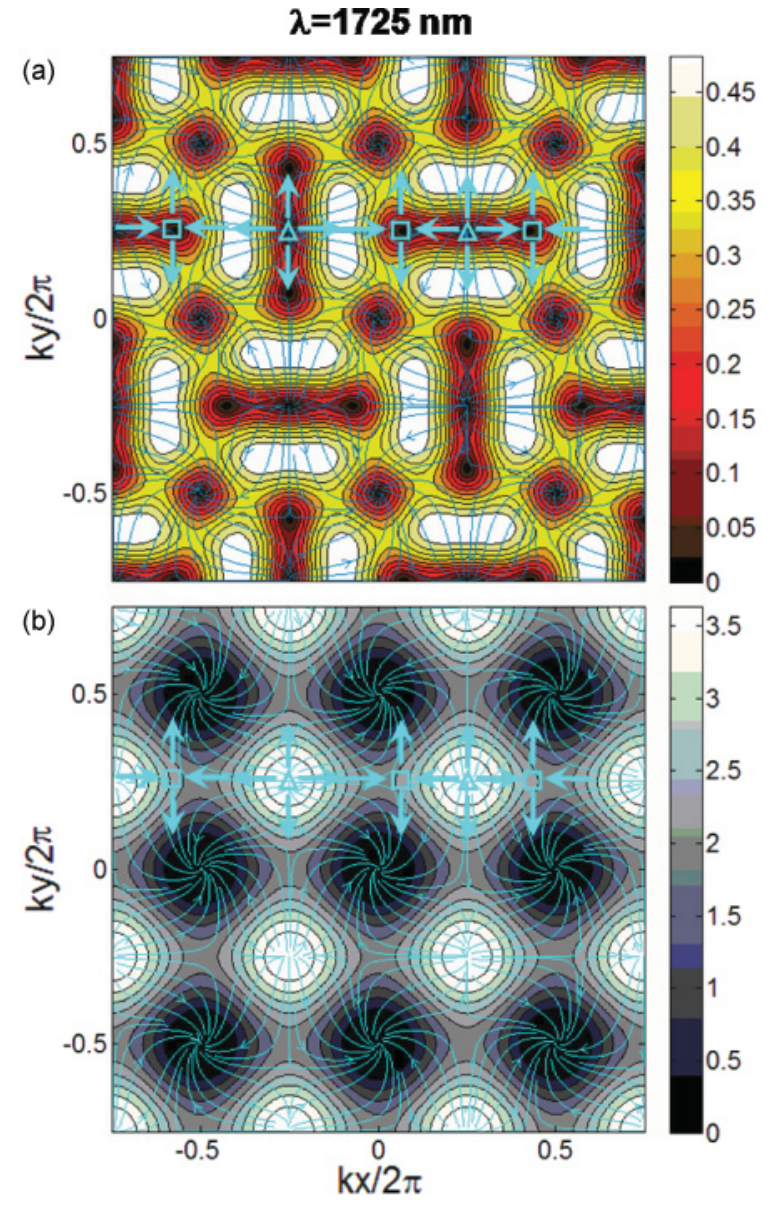

FIG. 7. (Color online) Same as Fig. 2 for a wavelength $\lambda=$ $1725 \mathrm{~nm}$ slightly above (red-shifted) the magnetic dipolar resonance. Although the force has both contributions, conservative and nonconservative, the conservative magnetic force dominates giving rise to stable equilibrium positions centered where the electric field is less intense. The equilibrium (zero-force) positions, corresponding to magnetic field maxima, are now located at the vortex center.

the conservative force and the stable equilibrium positions in the system become centered around the high-magnetic-field regions where the electric field is less intense (see Fig. 7). This is reflected in Fig. 3 where the stable equilibrium positions (below the magnetic resonance) become unstable zero-force positions (i.e., effective potential maxima) above the resonance. Interestingly, the vortices are now centered around the effective potential minima leading to vortex lines converging toward the centers.

Finally, let us mention some results concerning the case of zero dephasing. For dephasing $\phi=0$ so the force is always conservative. Interestingly, near the dipolar electric resonance the electric force is negligible and the effective potential landscape is entirely due to the magnetic and electric-magnetic interaction force contribution. The force map is shown in Fig. 8.

Finally, we should emphasize that it is possible to obtain equivalent results for $p$-polarization. In this case the electric force is always conservative and curl terms appear in the magnetic and electric-magnetic force contribution. The force maps near the magnetic resonances will now be similar to that 


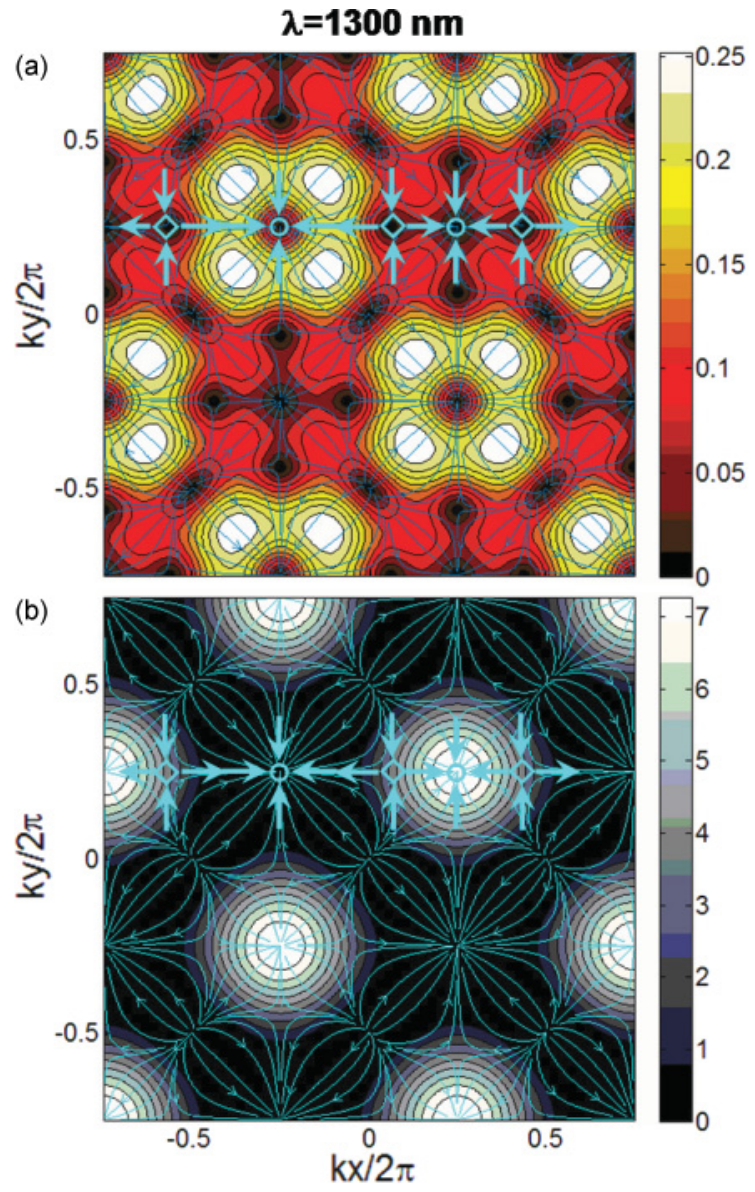

FIG. 8. (Color online) Same as Fig. 4 when the crossed beams oscillate synchronously $(\phi=0)$. At the dipolar electric resonance the electric forces are zero. The force field in this case is entirely given by magnetic or electric-magnetic forces.

discussed for $s$-polarized beams near the electric resonance and viceversa.

\section{CONCLUSION}

We have studied the optical forces on real small dielectric particles made of nonmagnetic materials with magnetodielectric response in optical vortex fields. We have demonstrated that those forces can be written as a sum of conservative and nonconservative components. Also, we have shown that the geometry of the force lines can be easily tailored by tuning both the wavelength and the phase shift between the fields forming the standing-wave pattern. The vortex-line forces depend on the both the chemical nature and the morphology of the particle through its complex polarizabilities. Submicron Si particles constitute an excellent laboratory to observe such new force effects. Similar effects are predicted for particles having large relative refractive indices such as $\mathrm{Ge}$ and $\mathrm{TiO} 2$ particles. Light vortex fields can offer a number of advantages to assess the local mechanical properties of cells and biological fluids [33]. In turn, the nonconservative forces may be used as a tunable probe of (nonlinear) properties of viscoelastic fluids or soft solids. The relevance of the electric-magnetic dipole interaction suggests possible applications in particle separation and sorting. We expect that these results will open new avenues in the controlled transport of polarizable small particles.

\section{ACKNOWLEDGMENTS}

This work has been supported by the Spanish MEC through the Consolider NanoLight (CSD2007-00046) and FIS2006-11170-C01-C02 and FIS2009-13430-C01-C02 research grants. Work by R.G.-M. financed by the MICINN "Juan de la Cierva" Grant.

\section{APPENDIX}

The three force terms of the decomposition Eq. (5), of the total magnetic force, resulting from the interference field given by Eq. (9), are expressed as

$$
\begin{aligned}
\left\langle\mathbf{F}_{\mathrm{m}}^{\langle U\rangle}\right\rangle= & \frac{4 \pi \operatorname{Re}\left\{\alpha_{\mathrm{m}}\right\}}{\epsilon} \nabla\left\langle U_{\mathrm{m}}\right\rangle=-\frac{\operatorname{Re}\left\{\alpha_{\mathrm{m}}\right\}}{2}\left|E_{0}\right|^{2} k \sin (2 k x) \hat{\mathbf{u}}_{x} \\
& -\frac{\operatorname{Re}\left\{\alpha_{\mathrm{m}}\right\}}{2}\left|E_{0}\right|^{2} k \sin (2 k y) \hat{\mathbf{u}}_{y}, \\
\left\langle\mathbf{F}_{\mathrm{m}}^{\langle S\rangle}\right\rangle= & \sigma_{\mathrm{m}}^{\mathrm{ext}}\left\{\frac{n}{c}\langle\boldsymbol{S}\rangle\right\}=-\frac{\sigma_{\mathrm{m}}^{\mathrm{ext}}}{4 \pi} \epsilon\left|E_{0}\right|^{2} \sin \phi \cos k x \sin (k y) \hat{\mathbf{u}}_{x} \\
& +\frac{\sigma_{\mathrm{m}}^{\mathrm{ext}}}{4 \pi} \epsilon\left|E_{0}\right|^{2} \sin \phi \cos k y \sin (k x) \hat{\mathbf{u}}_{y},
\end{aligned}
$$

where

$$
\left\{\frac{n}{c}\langle\boldsymbol{S}\rangle\right\}=\frac{\epsilon}{4 \pi k}\left|E_{0}\right|^{2} \nabla \times\left\{\sin \phi \cos k x \cos (k y) \hat{\mathbf{u}}_{z}\right\},
$$

and

$$
\begin{aligned}
\left\langle\mathbf{F}_{\mathrm{m}}^{\langle L\rangle}\right\rangle= & -\sigma_{\mathrm{m}}^{\mathrm{ext}}\left\{\frac{c}{n} \nabla \times\left\langle\boldsymbol{L}_{S \mathrm{~m}}\right\rangle\right\} \\
= & \sigma_{\mathrm{m}}^{\mathrm{ext}} \frac{\epsilon\left|E_{0}\right|^{2}}{4 \pi} \sin \phi \sin k y \cos (k x) \hat{\mathbf{u}}_{x} \\
& -\sigma_{\mathrm{m}}^{\mathrm{ext}} \frac{\epsilon\left|E_{0}\right|^{2}}{4 \pi} \sin \phi \sin k x \cos (k y) \hat{\mathbf{u}}_{y},
\end{aligned}
$$

where

$$
\left\langle\boldsymbol{L}_{S \mathrm{~m}}\right\rangle=\frac{\epsilon\left|E_{0}\right|^{2}}{4 \pi \omega} \sin \phi \cos k x \cos (k y) \hat{\mathbf{u}}_{z} .
$$

Notice that Eqs. (A4) and (A2) cancel each other when substituted into Eq. (5); namely, $\left\langle\mathbf{F}_{\mathrm{m}}^{\langle S\rangle}\right\rangle=-\left\langle\mathbf{F}_{\mathrm{m}}^{\langle L\rangle}\right\rangle$. Therefore the total magnetic force is always conservative and independent of the phase shift:

$$
\left\langle\boldsymbol{F}_{\mathrm{m}}\right\rangle=\left\langle\boldsymbol{F}_{\mathrm{m}}^{\langle U\rangle}\right\rangle+\left\langle\boldsymbol{F}_{\mathrm{m}}^{\langle S\rangle}\right\rangle+\left\langle\boldsymbol{F}_{\mathrm{m}}^{\langle L\rangle}\right\rangle=\left\langle\boldsymbol{F}_{\mathrm{m}}^{\langle U\rangle}\right\rangle .
$$

[1] P. Reiman, Phys. Rep. 361, 57 (2002).

[2] T. G. Mason, K. Ganesan, J. H. vanZanten, D. Wirtz, and S. C. Kuo, Phys. Rev. Lett. 79, 3282 (1997).
[3] F. Scheffold and P. Schurtenberg, Soft Mater 1, 139 (2003).

[4] V. M. Rotello, Adv. Drug Delivery Rev. 60, 1255 (2008).

[5] A. Ashkin, Proc. Natl. Acad. Sci. USA 94, 4853 (1997). 
[6] J. E. Curtis, B. A. Koss, and D. G. Grier, Opt. Comm. 207, 169 (2002).

[7] M. M. Burns, J.-M. Fournier, and J. A. Golovchenko, Science 249, 749 (1990).

[8] M. I. Antonoyiannakis and J. B. Pendry, Europhys. Lett. 40, 613 (1997).

[9] R. Gómez-Medina, P. SanJose, A. Garcia-Martin, M. Lester, M. Nieto-Vesperinas, and J. J. Saenz, Phys. Rev. Lett. 86, 4275 (2001).

[10] P. T. Korda, M. B. Taylor, and D. G. Grier, Phys. Rev. Lett. 89, 128301 (2002).

[11] M. P. MacDonald, G. C. Spalding, and K. Dholakia, Nature (London) 426, 421 (2003).

[12] M. M. Burns, J.-M. Fournier, and J. A. Golovchenko, Phys. Rev. Lett. 63, 1233 (1989).

[13] S. A. Tatarkova, W. Sibbett, and K. Dholakia, Phys. Rev. Lett. 91, 038101 (2003).

[14] R. Gómez-Medina and J. J. Sáenz, Phys. Rev. Lett. 93, 243602 (2004).

[15] M. Guillon, O. Moine, and B. Stout, Phys. Rev. Lett. 96, 143902 (2006).

[16] S. Gaugiran, S. Gétin, J. M. Fedeli, and J. Derouard, Opt. Express 15, 8146 (2007).

[17] K. Dholakia and P. Zemánek, Rev. Mod. Phys. 82, 1767 (2010).

[18] P. C. Chaumet and M. Nieto-Vesperinas, Phys. Rev. B 61, 14119 (2000); P. C. Chaumet, A. Rahmani, and M. Nieto-Vesperinas, Phys. Rev. Lett. 88, 123601 (2002); M. Nieto-Vesperinas, P. C. Chaumet, and A. Rahmani, Philos. Trans. R. Soc. London A 362, 719 (2004).
[19] B. A. Kemp, T. M. Grzegorczyk, and J. A. Kong, Phys. Rev. Lett. 97, 133902 (2006).

[20] M. Mansuripur, Opt. Express 12, 5375 (2004).

[21] S. Albaladejo, M. I. Marques, M. Laroche, and J. J. Sáenz, Phys. Rev. Lett. 102, 113602 (2009).

[22] P. C. Chaumet and A. Rahmani, Opt. Express 17, 2224 (2009)

[23] M. Nieto-Vesperinas, J. J. Sáenz, R. Gómez-Medina, and L. Chantada, Opt. Express 18, 11428 (2010).

[24] P. Zemánek, V. Karásek, and A. Sasso, Opt. Commun. 240, 401 (2004).

[25] S. Albaladejo, M. I. Marques, F. Scheffold, and J. J. Sáenz, Nano Lett. 9, 3527 (2009).

[26] I. Zapata, S. Albaladejo, J. M. R. Parrondo, J. J. Saenz, and F. Sols, Phys. Rev. Lett. 103, 130601 (2009).

[27] A. Hemmerich and T. W. Hänsch, Phys. Rev. Lett. 68, 1492 (1992); 70, 410 (1993).

[28] P. Zemánek, M. Šiler, V. Karásek, and T. Čižmár, Proc. SPIE Int. Soc. Opt. Eng. 5930, 59301N (2005).

[29] A. García-Etxarri et al., Opt. Express 19, 4815 (2011).

[30] G. Grynberg, B. Lounis, P. Verkerk, J. Y. Courtois, and C. Salomon, Phys. Rev. Lett. 70, 2249 (1993).

[31] H. C. Hulst, Light Scattering by Small Particles (Dover, New York, 1981).

[32] M. Nieto-Vesperinas, R. Gómez-Medina, and J. J. Sáenz, J. Opt. Soc. Am. A 28, 54 (2011).

[33] J. C. Crocker and B. D. Hoffman, Methods Cell Biol. 83, 141 (2007). 